\title{
A Study of Phase Angle Distribution of Partial Discharges in Oil Pressboard Insulation Systems
}

\author{
K. Raja, M.N. Narayanachar and R.S. Nema \\ Department of High Voltage Engineering \\ Indian Institute of Science \\ Bangalore-560 012, India
}

\begin{abstract}
In electrical power transformers, composite insulation consisting of oil-pressboard insulation is widely used. For optimum design of such insulation systems, partial discharge (PD) behaviour is an important design criterion. A computer aided PD measuring system which per. mits a time sequential data acquisition and phase resolved pulse height analysis has been developed. Using this system, PD in a representative oilpressboard system has been measured. The ef.fect of stress level, their duration and the test procedure on the discharges have been studied. The discharge data is analysed using Gumbel and Weibull statistics. An attempt is made to correlate the Weibull and derived PD parameters of each phase with their counter-parts in the cycle.
\end{abstract}

\section{INTRODUCTION}

Pressboard combined with mineral oil often constitutes the main insulation of high voltage transformers. It is well known that PD in a oilpressboard insulation system is one of the main causes of deterioration in the insulation system. This work is aimed at studying the PD characteristics of a oil-pressboard system, which consists of a impregnated pressboard disc held between two Rogowski profile electrodes. PD data are recorded according to their phase of occurrence with respect to the power frequency cycle, their magnitude and number over a period of time. The results are analysed using Gumbel and Weibull statistics and Spearman's rank correlation test statistic.

\section{EXPERIMENTAL SET-UP}

A block diagram of the phase resolved partial discharge analyser (PRPDA) including PD measuring circuit is shown in Figure 1. The system consists of a measuring impedance $\mathrm{Zm}$, narrow band tuned amplifier, pulse shaping unit, pulse height analyser and personal computer, including software for data storage and processing. The ac cycle $\left(360^{\circ}\right)$ is split into 16 phase windows each covering $22.5^{\circ}$ and the pulses in each window are sorted out into 512 magnitude windows and counted. The high voltage test circuit, has been found to be discharge free upto $70 \mathrm{kV}$ with a sensitivity of $2 \mathrm{pc}$.

\section{SAMPLE PREPARATION AND EXPERIMENTAL PROCEDURE}

Pressboard samples of nominal thickness 2.1 $\mathrm{mm}$ were cut from big sheets and finished on the lathe to $50 \mathrm{~mm}$ diameter. Care was taken to see that there were no visual burrs at the edges and the finishing was smooth. $7 \pi / 12$ Rogowski profile uniform field brass electrodes of $30 \mathrm{~mm}$ overall diameter were used and the impregnated sample is placed in between the electrodes inside a perspex tank filled with transformer oil. The oil was filtered periodically using G3 and G4 sintered glass filters and dried under vacuum of 0.1 torr for about 2 hours. The oil was then allowed to 
be saturated with dry air for about 8 hours and this oil was used for experiments. The breakdown voltage of oil was in the range of 40 to $45 \mathrm{kV}$ for a $1 \mathrm{~mm}$ gap between $12.5 \mathrm{~mm}$ spheres.

It was observed during preliminary experiments, that PD spectrum depended upon the level of stress, its duration and number of steps of voltage application. To study these effects, the experiments were done at different overvoltages and stress level durations. To start with, the PD inception voltage $\left(\mathrm{V}_{i}\right)$ and surface flashover voltage $\left(V_{f}\right)$ of about 16 samples were determined. To find $\mathrm{V}_{i}$, the voltage was slowly raised at a rate of $0.5 \mathrm{kV} / \mathrm{sec}$ till discharges persisting for atleast 1 minute were observed. To determine $V_{f}$, the rate of rise of voltage was $2 \mathrm{kV} / \mathrm{sec} . \mathrm{V}_{i}$ and $\mathrm{V}_{f}$ were in the range of 30 to $37 \mathrm{kV}$ and 55 to $60 \mathrm{kV}$ respectively.

The samples were aged at $V_{i}$ and in steps of 5 and 10 percent of $V_{i}$. Step-size and its duration was based upon the discharge spectrum observed on the oscilloscope. If the discharges were intermittent and if the number density and discharge magnitude were insignificant, then the next higher step of voltage was applied. It was found that at one particular voltage step, the discharge magnitude, $\mathrm{Q}$ and pulse rate $\left(\mathrm{N}_{t}\right)$ suddenly increased and persisted in all phases. The voltage of this stage is kept constant till the sample failed.

\section{TEST RESULTS AND ANALY- SIS}

The results of the investigations and the analysis of a typical sample is presented in this section. In this experiment, $\mathrm{V}_{i}$ was $32 \mathrm{kV}$ and the sample was aged at $V_{i}$ for 6 hours following which the voltage was raised to $1.1 \mathrm{~V}_{i}$ and kept constant for about 3 hours. The last step of ageing was $1.15 \mathrm{~V}_{i}$ and it lasted for 1.12 hours when sample failed due to puncture. Fig. 2 depicts phase resolved parameter distribution (PRPD) plots of total charge $\left(Q_{t}\right)$ and maximum charge $\left(Q_{m}\right)$ as a function of ageing time. A study of PRPD plots reveals that initial period of sample ageing is characterized by the intermittent appearance of pulses, poor pulse rate ( 0.01 to 0.03 per sec.) and scarce distribution of pulses amongst the phases. Maximum PD magnitude was less than 15 pc. Prior to sample failure, luminous sparks of discharges were seen at the upper and lower interfaces of the sample surface with the electrodes. The start of this event is characterized by the appearance of copious PD pulses of various magnitudes ranging from $3 \mathrm{pc}$ to $150 \mathrm{pc}$. Sudden shoot in all diagnostic parameters $\left(\mathrm{N}_{t}, \mathrm{Q}_{t}, \mathrm{Q}_{m}\right)$ were observed for all phases. The factor of increase in $\mathrm{N}_{t}$ ranged between 60 to $550, \mathrm{Q}_{t}$ between 50 to 180 , and $\mathrm{Q}_{m}$ between 5 to 12 . This mode of appearance and mushrooming existence of discharges in all phases is termed burst discharges. After the commencement of burst discharges, the sample failed in about 29 minutes. A visual examination of failed sample reveal the presence of marks of discharges tracing a circular path on the sample surface. The puncture occurred on the circumference of this path.

PRPD plot of phase window wise distribution, clearly show that phase windows $\Phi_{14}$ and $\Phi_{16}$ contribute significantly to the total $\mathrm{Q}_{t}$. Their contributions are respectively 25.2 and 19.4 percent respectively. $\Phi_{7}$ is the least contributor, followed by $\Phi_{8}$ and their respective contributions are 0.5 and 1.3 percent respectively. Similar observations were also seen for PRPD plot of $N_{t}$ (not shown in figure.). These observations were consistent for all experiments performed using the test procedure detailed above. Also, for this experiment, it was observed that $Q_{t}$ of negative half cycle is about 84.3 percent of total $Q_{t}$. PRPD plot of $Q_{m}$ show that $\Phi_{7}$ and $\Phi_{8}$ records the least $Q_{m}$. The maximum values observed in these phases are respectively 37.1 and 54.6 percent of the maximum $\mathrm{Q}_{m}$ observed during the experiment. This is also substantiated by low values of Weibull $\alpha$ and $\beta$ obtained in these phases.

\section{STATISTICAL ANALYSIS AND DISCUSSION}

Pre-burst discharge data in individual phases 
were insufficient for distribution fitting of discharge spectrum. To characterize this ageing period, $Q_{m}$ of the $\mathrm{PD}$ data collected at regular intervals of time for different phases and cycle were arranged as a sequence of time-series records ('TSR). Gumbel's extreme value distribution of largest element [1] given by,

$$
\mathrm{F}(\mathrm{q})=\exp \{-\exp (-[\mathrm{q}-\mu] / \sigma)\}
$$

was fitted to the TSR of $\mathrm{Q}_{m}$. The parameters $\mu$ and $\sigma$ are respectively the location and scale: parameters, $\mathrm{q}$ is the discharge magnitude, $\mu$ and $1.283 \sigma$ are respectively the mode and standard deviation of the distribution. Their values for the complete cycle are respectively $6.55 \mathrm{pc}$ and 2.39 .

Post-burst discharge data was observed to follow a 3- parameter Weibull distribution [1] given by,

$$
\mathrm{F}(\mathrm{q})=1-\exp ((-\mathrm{q}-\gamma) / \alpha)^{\beta}
$$

where $\alpha$ is the scale parameter which represents the discharge magnitude, below which lie $63.2 \%$ of the discharge magnitudes. $\beta$ is a shape parameter which is a measure of the dispersion of the discharge magnitudes. $\gamma$ is the location parameter indicating the discharge magnitude below which no discharges occurs. TSR of $\alpha, \beta, \mathrm{N}_{t}$ and $Q_{t}$ were obtained as before and Spearman's rank correlation technique [2] was used to study the degree of correlation between the respective phase and cycle TSR of Weibull and diagnostic parameters. 'Iable 1 presents the results of the correlation at significance level, $\sigma=0.1$. Column $\Phi_{14}$ of the table shows that there exists a definite correlation between all parameters studied. Blanks (-) in the table mean that there is no significant correlation.

\section{REFERENCES}

1. Hahn, G.J. and Shapiro, S.S. Statistical Models in Engineering, John Wiley \& Sons, New York, 1967.

2. Conover, W.J. Practical Non-parametric Statistics, John Wiley \& Sons, New York, 1980.

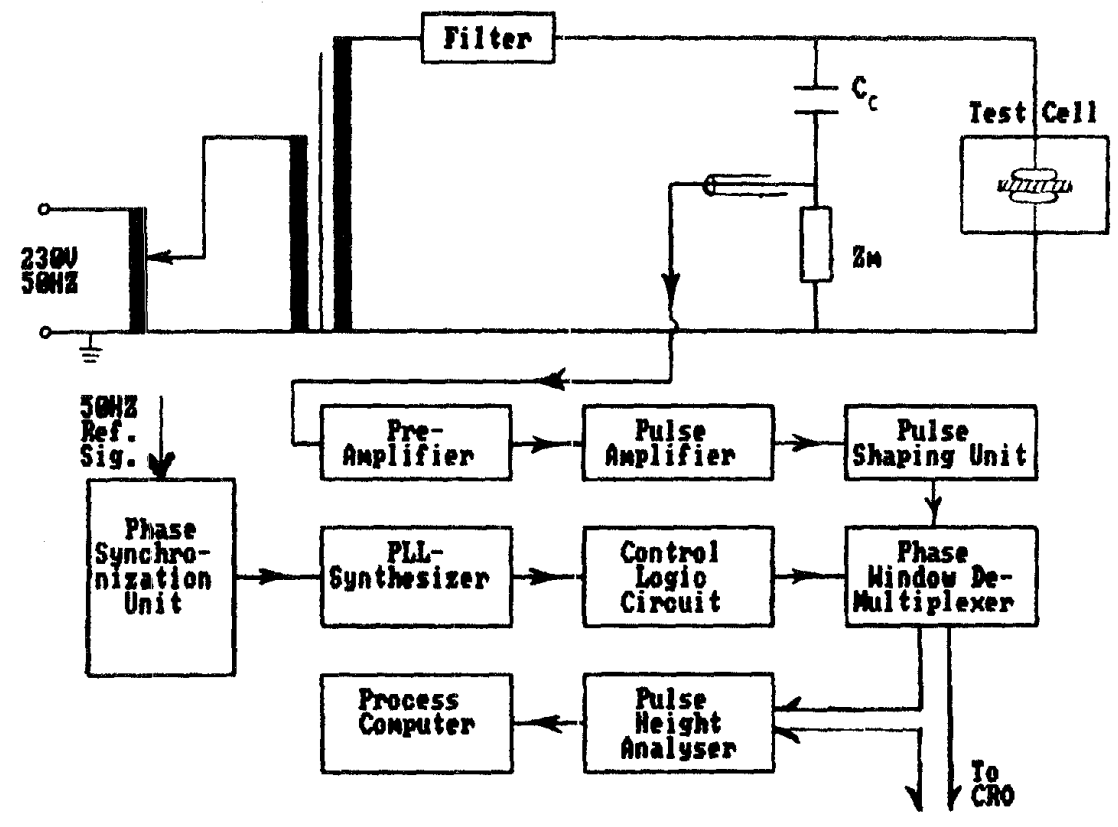

Figure 1. Block diagram of experimental set-up 

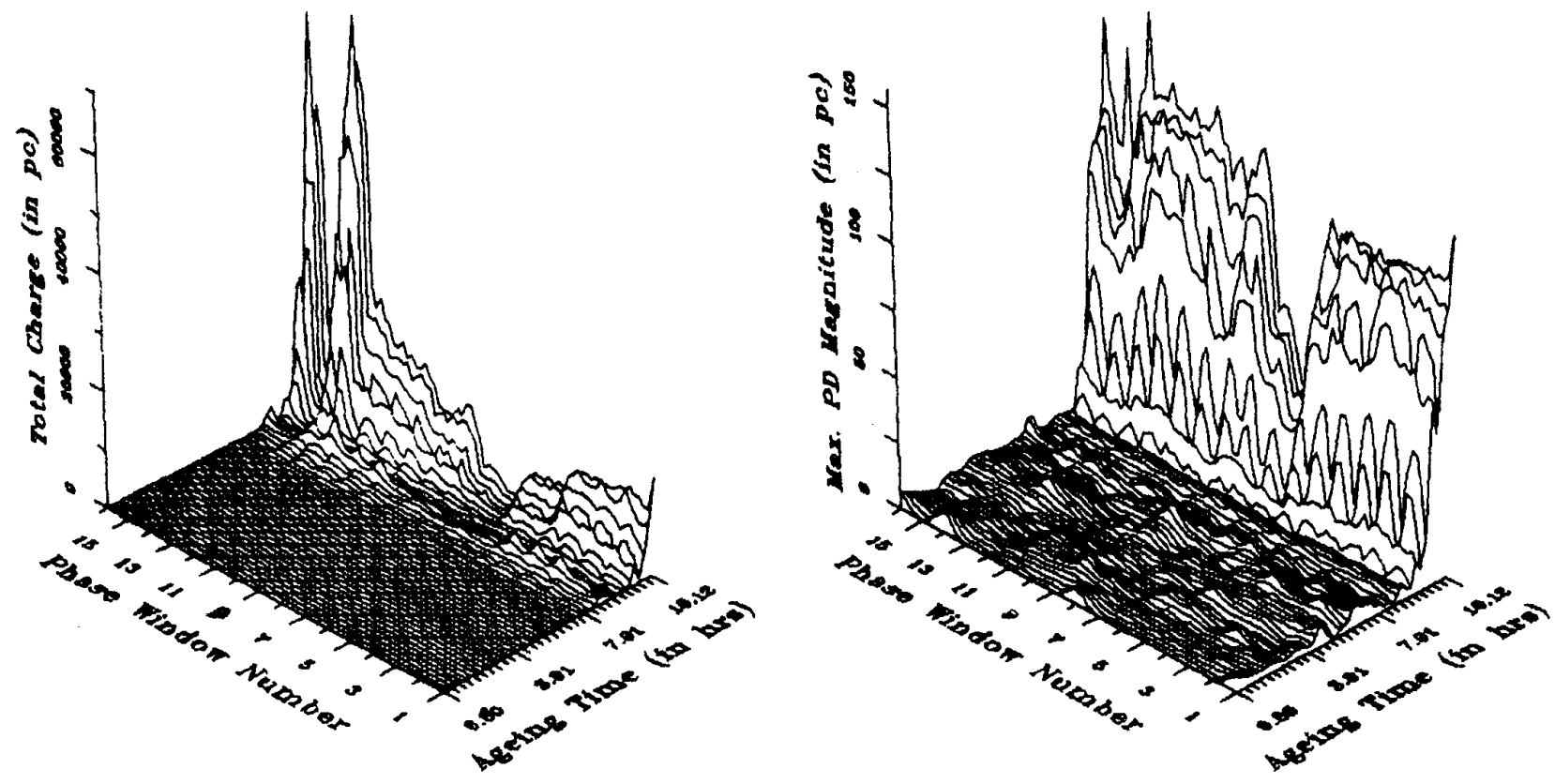

Figure 2. $P R P D$ plot of $Q_{t}$ and $Q_{m}$

Table 1

\begin{tabular}{|c|c|c|c|c|c|c|c|c|c|c|c|c|c|c|c|c|}
\hline Parameter & $\overline{\phi_{1}}$ & $\phi_{2}$ & $\phi_{3}$ & $\phi_{1}$ & $\phi_{5}$ & $\phi_{6}$ & $\phi_{7}$ & $\overline{\phi_{8}}$ & $\phi_{9}$ & $\phi_{10}$ & $\phi_{11}$ & $\phi_{12}$ & $\phi_{13}$ & $\phi_{14}$ & $\phi_{15}$ & $\phi_{16}$ \\
\hline$\alpha_{\phi}$ Vs $\alpha_{c y c l e}$ & 0.60 & 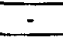 & $=$ & - & 0.65 & - & 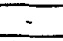 & - & 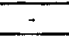 & $=$ & 0.50 & 0.52 & & 0.57 & . & 0.70 \\
\hline$\beta_{\phi} V_{s} \beta_{\text {cycle }}$ & & - & 0.62 & - & - & - & 0.73 & - & 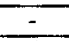 & - & 0.72 & 0.55 & 0.65 & 0.58 & & 0.68 \\
\hline$N_{t, \phi} V_{s} N_{t, c y c l e}$ & 0.82 & 0.85 & 0.95 & 0.85 & 0.83 & 0.82 & 0.77 & 0.57 & 0.85 & 0.98 & 0.85 & 0.85 & 0.80 & 0.98 & 0.88 & 0.95 \\
\hline$Q_{t, \phi} V_{s} Q_{t, c y c l e}$ & 0.80 & 0.87 & 0.88 & 0.85 & 0.78 & 0.78 & 0.72 & 0.68 & 0.81 & 0.85 & 0.97 & 0.90 & 0.88 & 0.98 & 0.92 & 0.95 \\
\hline$\alpha_{\phi} V_{s} N_{t, \phi}$ & 0.83 & 0.87 & $=$ & 0.62 & 0.88 & 0.48 & - & - & 0.72 & 0.62 & 0.68 & - & 0.57 & 0.70 & 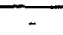 & 0.52 \\
\hline$\beta_{\phi} V_{s} N_{t, \phi}$ & 0.63 & 0.77 & 0.45 & 0.70 & 0.53 & - & $\therefore$ & $=$ & 0.57 & 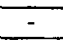 & - & - & 0.47 & 0.72 & - & 0.77 \\
\hline$\alpha_{\phi} V_{s} Q_{t, \phi}$ & 0.95 & - & 0.85 & - & $=$ & 0.53 & 0.50 & - & 0.77 & 0.72 & 0.57 & - & 0.57 & $0.7 \overline{7}$ & - & 0.58 \\
\hline$\beta_{\phi} v_{s} Q_{t, \phi}$ & 0.70 & - & - & 0.83 & 0.48 & - & $=$ & - & - & 0.53 & $=$ & 7 & 0.47 & $0 . \overline{73}$ & - & 0.60 \\
\hline$\alpha_{\phi} V_{s} \bar{\beta}_{\phi}$ & 0.77 & 0.97 & - & 0.67 & 0.55 & 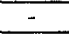 & - & 0.78 & 0.77 & 0.75 & 0.82 & 0.93 & 0.93 & 0.63 & 0.85 & - \\
\hline
\end{tabular}

